\title{
Identification of long non-coding RNAs biomarkers associated with progression of endometrial carcinoma and patient outcomes
}

\author{
Yanan Sun ${ }^{1, *}$, Xiaoyan Zou ${ }^{1, *}$, Jun $\mathrm{He}^{1}$ and Yuqin Mao ${ }^{1}$ \\ ${ }^{1}$ Department of Gynecology and Obstetrics, Daqing Oilfield General Hospital, Daqing 163000, China \\ *These authors contributed equally to this work
}

Correspondence to: Yanan Sun, email: ynsundq@163.com

Keywords: biomarkers, endometrial carcinoma, long non-coding RNA

Received: March 13, 2017

Accepted: April 07, 2017

Published: April 30, 2017

Copyright: Sun et al. This is an open-access article distributed under the terms of the Creative Commons Attribution License 3.0 (CC BY 3.0 ), which permits unrestricted use, distribution, and reproduction in any medium, provided the original author and source are credited.

\section{ABSTRACT}

Endometrial carcinoma is a complex disease characterized by both genetic, epigenetic and environmental factors. Increasing evidence has suggested that long non-coding RNAs (IncRNAs) play important roles in the development and progression of cancers. In this study, we performed a comparison analysis for IncRNA expression between patients with early-stage (stage I/II) and those with advanced-stage (stage III/IV) derived from The Cancer Genome Atlas (TCGA) project and identified 17 differentially expressed IncRNAs using student t-test. Five of the 17 differentially expressed IncRNAs were selected as optimal biomarkers that are significantly associated with progression of UCEC using random forest feature selection procedure. A risk classifier of five IncRNAs was developed to as a molecular signature that identifies patients at high risk for progression using support vector machine. Results of fiveIncRNA risk classifier achieved high discriminatory performance in distinguishing advanced stage from early stage with $78 \%$ prediction accuracy, $96.6 \%$ sensitivity and $76.6 \%$ specificity. Functional analysis suggested that these five IncRNA biomarkers may play critical roles in the progression of UCEC by participating in important cancer-related biological processes. Our study will help to improve our understanding of underlying mechanisms in the progression of UCEC and provide novel IncRNAs as candidate predictive biomarkers for the identification of patients with high risk for progression.

\section{INTRODUCTION}

Endometrial carcinoma is the most common malignancy in the female population with a rapidly increasing trend worldwide [1]. Although the outcome is favorable for many cases diagnosed at an early stage with a five-year survival rate of $75 \% \sim 86 \%$ [2], some will relapse and eventually die. Treatment of endometrial cancer is dependent on the stage of the disease and surgical intervention, if possible, is the standard management. The majority of endometrial carcinoma patients with early stage will be cured with surgery alone. Adjuvant therapy (including radiation therapy and/or chemotherapy) after surgical intervention is another treatment option in cases of high-risk or advanced endometrial carcinoma patients and has been shown to improve survival in patients with advanced stage [3]. However, the fact that a subgroup of patients with early stage faced an increased risk of cancer progression and recurrence, has led to an urgent need to identify predictive biomarkers that help clinicians determine which patients with early-stage might benefit from more aggressive therapy.

The sequencing of the human genome has suggested that only $<2 \%$ of the total genomic sequence encodes only $\sim 20,000$ protein coding genes, whereas most of the human genome can be transcribed, yielding tens of thousands of non-coding RNAs (ncRNA) [4, 5]. NcRNAs are grouped into two major categories based on transcript size: small ncRNAs and long non-coding RNAs (lncRNAs). LncRNAs, representing the major class of ncRNAs, was arbitrarily defined as mRNA-like transcripts ranging in length from 200 nucleotides (nt) to $\sim 100$ kilobases $(\mathrm{kb})$ lacking significant protein-coding capacity [6]. A large number of studies have shown that lncRNAs play a critical role in various fundamental biological processes by regulating gene expression at 
epigenetic, transcriptional, post-transcriptional levels [7-9]. Aberrant lncRNA expression has widely been observed in various cancers [10-12]. It is becoming increasingly apparent that these dysregulated lncRNAs are specifically associated with the development and progression of cancers [13-18]. Some well-characterized IncRNAs have been found to possess oncogenic or tumor suppressive roles and function as a biomarker for cancer diagnosis or prognosis $[19,20]$, such as H19, HOTAIR, MALAT-1, HULC and so on. However, the lncRNA biomarkers for EC progression has not been previously explored.

In this study, we investigated lncRNA expression profiles in a large cohort of patients with uterine corpus endometrial carcinoma (UCEC) and attempted to identify lncRNAs capable of identifying patients at high risk for progression to advanced UCEC as novel clinical predictive biomarkers.

\section{RESULTS}

\section{Identification of deregulated IncRNA expression during the progression of UCEC}

We first investigated whether there was altered IncRNA expression pattern during the progression of UCEC by comparing IncRNA expression profiles of advanced-stage patients with those with early-stage. A total of 17 lncRNAs were differentially expressed between patients with advanced-stage and those with early-stage using $T$-test with a false discovery rate $(\mathrm{FDR})<0.01$ after Benjamini \& Hochberg correction and t-statistic $>4$ (or < -4) (Supplementary Table 1). Among them, 14 lncRNAs were down-regulated and three lncRNAs were up-regulated in patients with advanced stage compared with those with early stage (Figure 1A).

Then we clustered 300 UCEC patients according to the expression level of differentially expressed 17 IncRNAs which resulted in two distinctive patient clusters (Figure 1B). The results of chi-square test showed that the disease progression state of the two patient clusters was significantly different ( $p<0.001$, chi-square test). Specifically, Cluster 1 contained close to the majority of advanced-stage patients $(n=54 ; 70.1 \%)$. Conversely, Cluster 2 contained the majority of early-stage patients $(n=164 ; 73.5 \%)$. The Kaplan-Meier analysis and logrank test revealed that the overall survival time between the two patient clusters was significantly different ( $p=0.023$, log-rank test) (Figure 2). At three and five years, the survival rates of UCEC patients in Cluster 1 were $84.6 \%$ and $68.8 \%$, respectively, whereas the corresponding rates in the Cluster 2 were $89.7 \%$ and $89.7 \%$, respectively. The above results demonstrated that these 17 altered lncRNAs might serve as predictive biomarkers for the identification of patients with high risk for progression.

\section{Identification of optimal predictive IncRNA biomarkers of UCEC progression}

To identify optimal predictive lncRNA biomarkers capable of identifying patients at high risk for progression to advanced stage, we performed feature selection and classification procedure using support vector machine and random forest method as described in Materials and methods. All differentially expressed lncRNAs were ranked according to the standardized drop in prediction accuracy as shown in Figure 3A. Then we compared diagnostic odds ratio (DOR) increment for a specific number of lncRNAs by subsequently adding one lncRNA at a time in a top-down forward-wrapper approach starting with the top two lncRNAs of the ranked list and identified five lncRNAs as a balance between classification accuracy and the number of lncRNAs. When choosing more than five lncRNAs, there is a downward trend in predictive performance (Figure $3 \mathrm{~B}$ ). Therefore, top five lncRNAs (FLJ27354, RP11-275I14.4, VIM-AS1, $C T B-51 J 22.1$ and $R P 11-229 P 13.20)$ in the ranked list were selected as optimal predictive lncRNA biomarkers of UCEC progression (Table 1). Among them, one lncRNAs (FLJ27354) tended to be active and the remaining four lncRNAs (RP11-275I14.4, VIM-AS1, CTB-51J22.1 and $R P 11-229 P 13.20)$ were silent in the progression of UCEC.

\section{Performance evaluation of five IncRNA biomarkers for UCEC progression}

To test whether selected optimal five lncRNA biomarkers could efficiently distinguish high-risk patients from low-risk patients, we performed unsupervised hierarchical clustering for 300 UCEC patients according to the expression values of five lncRNA biomarkers. The results of hierarchical clustering showed that all patients were grouped into two distinctive patients clusters (162 samples in Cluster 1 vs. 138 samples in Cluster 2), which were highly correlated with disease progression status ( $p<0.001$, Fisher exact test; Figure 4A). As seen in Figure 4A, most of the advanced patients $(71.4 \%$, 55/77) were clustered into Cluster 2 and most of the early patients $(62.8 \%, 140 / 223)$ were clustered into Cluster 1. Furthermore, the Kaplan-Meier analysis for overall survival demonstrated a significant difference between the groups predicted to be high-risk or low-risk ( $p=0.001$, log-rank test; Figure 4B). At three and five years, the survival rates of UCEC patients in the predicted high-risk group were $80.9 \%$ and $68.1 \%$, respectively, whereas the corresponding rates in the predicted low-risk group were both $93.9 \%$, respectively. These results revealed the better predictive performance of five lncRNAs biomarkers for the identification of patients with high risk for progression.

Thus, we integrated these five lncRNA biomarkers to construct a five-lncRNA risk classifier by using SVM algorithm. The performance of the five-lncRNA risk 
Table 1: Five IncRNA biomarkers associated with the progression of UCEC

\begin{tabular}{llllll}
\hline Ensembl id & Gene name & Chromosomal location & t-statistic & p-value & FDR \\
\hline ENSG00000231999.2 & FLJ27354 & Chr1: 89,583,241-89,632,894(-) & 4.35 & $3.63 \mathrm{E}-05$ & 0.00385 \\
ENSG00000234478.1 & RP11-275114.4 & Chr1: 226,148,003-226,155,071(+) & -4.06 & $6.28 \mathrm{E}-05$ & 0.00541 \\
ENSG00000229124.1 & VIM-AS1 & Chr10: 17,214,239-17,229,985(-) & -5.04 & $1.19 \mathrm{E}-06$ & 0.00041 \\
ENSG00000232415.1 & CTB-51J22.1 & Chr7: 74,059,576-74,062,284(-) & -4.3 & $2.39 \mathrm{E}-05$ & 0.00274 \\
ENSG00000235117.1 & RP11-229P13.20 & Chr9: 137,037,040-137,037,955(+) & -4.53 & $1.01 \mathrm{E}-05$ & 0.00175 \\
\hline
\end{tabular}
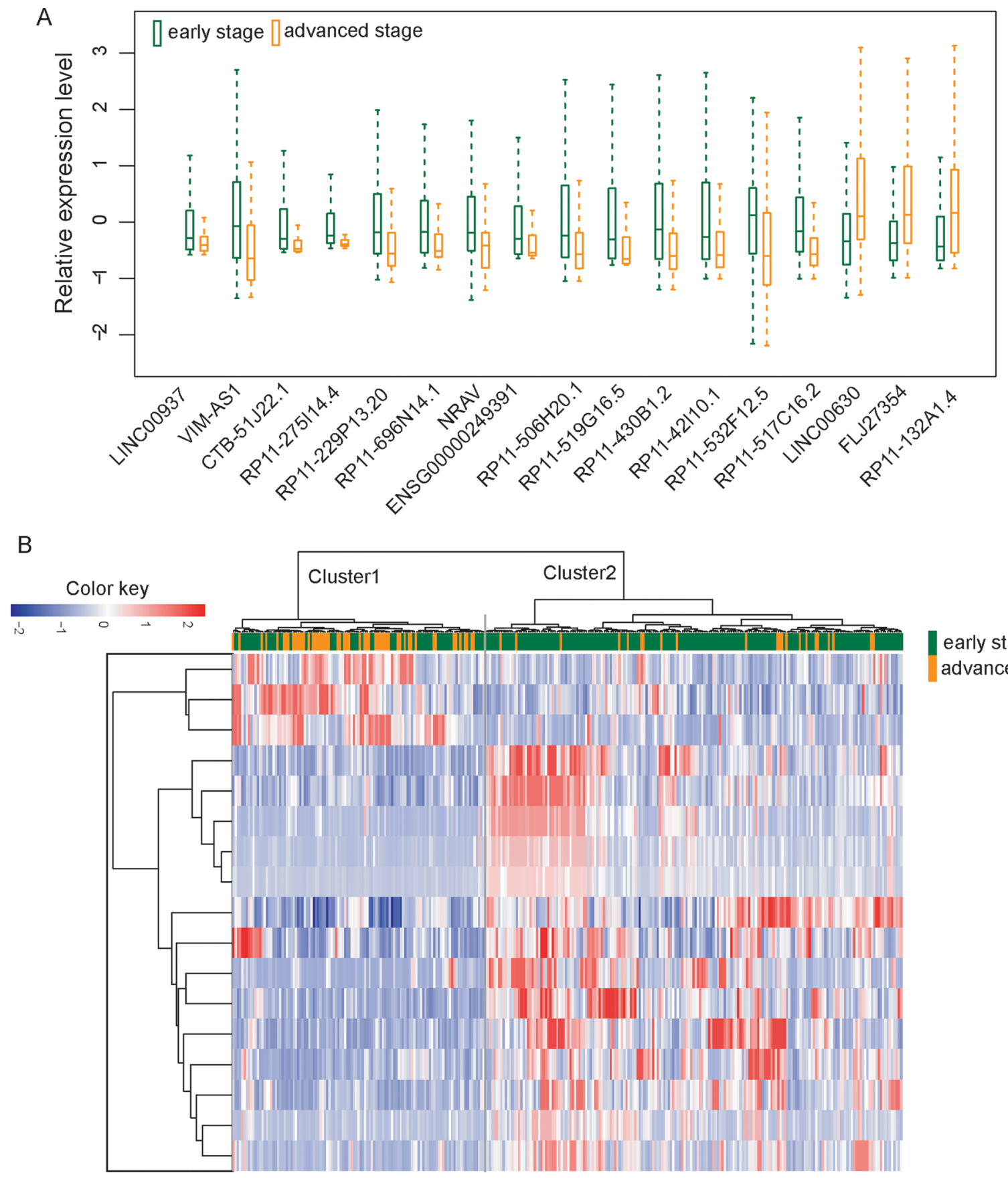

Figure 1: Altered IncRNA expression patterns in the progression of UCEC. (A) Boxplots of 17 differentially expressed lncRNAs between patients with advanced-stage and those with early-stage. (B) The unsupervised hierarchical clustering heatmap of 300 UCEC samples based on the expression profiles of 17 differentially expressed lncRNAs 
classifier in distinguishing advanced-stage UCEC patients from early-stage patients was evaluated in the TCGA cohort using the leave one out cross-validation (LOOCV) procedure, in which 299 patients were used as training set and the remaining one was served as the test patient. Results of LOOCV procedure showed that the fivelncRNA risk classifier for distinguishing advanced-stage patients from early-stage patients achieves $78 \%$ prediction accuracy with $96.6 \%$ sensitivity and $76.6 \%$ specificity. The discriminatory performance of the five-lncRNA risk classifier, evaluated by calculating the receiver operating characteristic curve (AUC) and DOR, revealed that the
AUC was 0.695 (Figure 4C) and the DOR was 9.4. These results demonstrated that the five-lncRNA risk classifier had the better predictive performance for identifying patients at risk for UCEC progression.

\section{Functional implication of five IncRNA biomarkers}

To explore the potential functional role of five lncRNA biomarkers in the progression of UCEC, we first examined the expression correlation between each of five lncRNA biomarkers and mRNAs in the TCGA

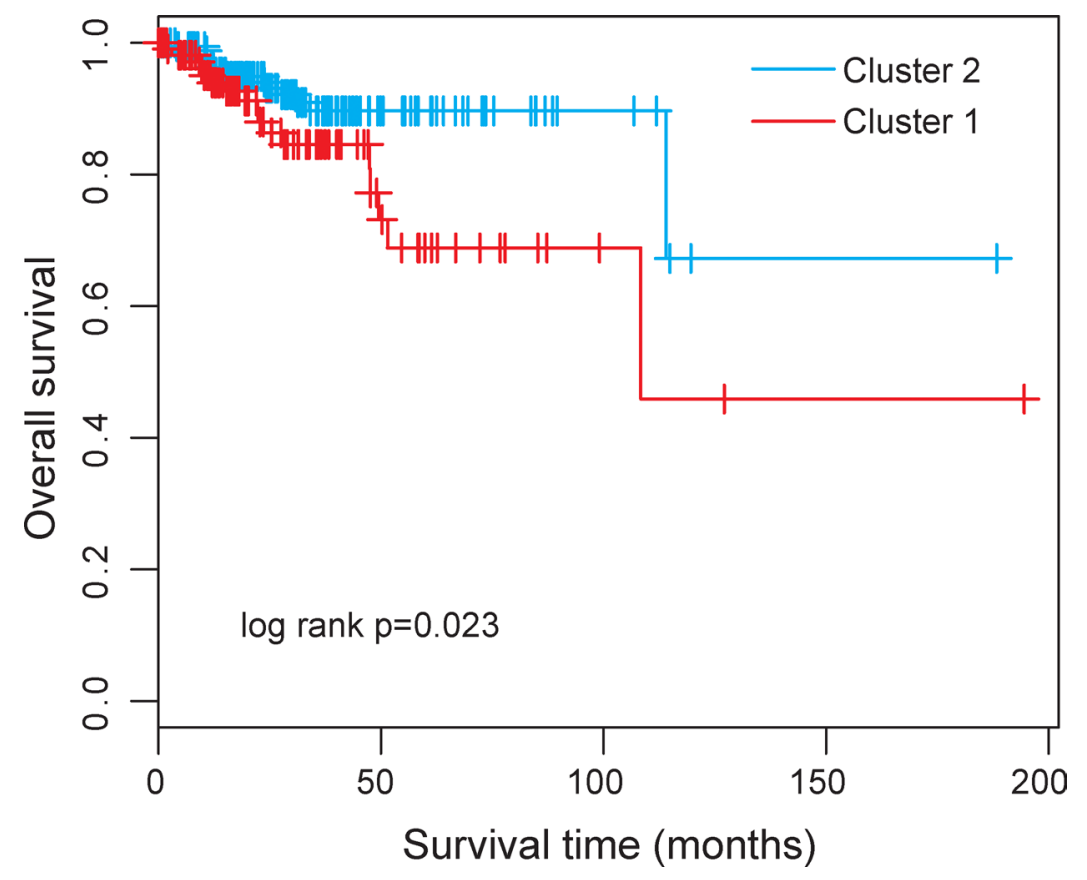

Figure 2: Kaplan-Meier survival curve for overall survival between the predicted two clusters based on 17 differentially expressed IncRNAs.
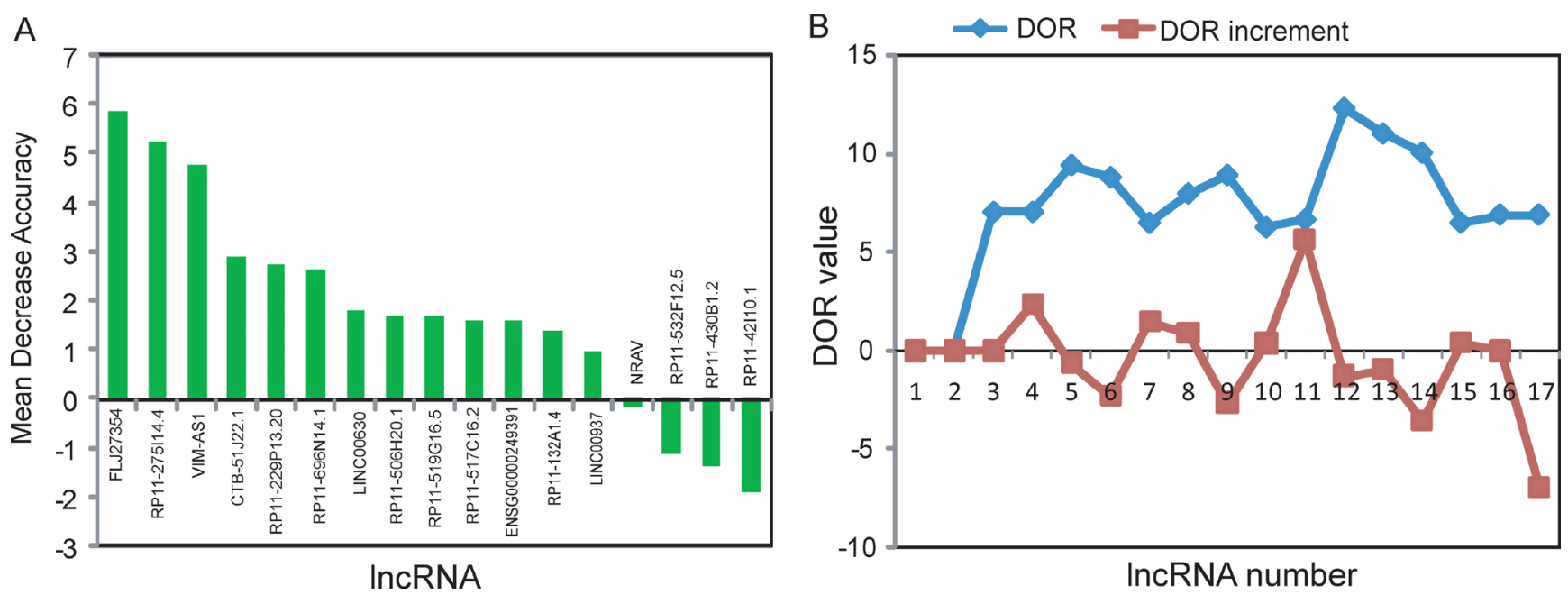

Figure 3: Identification of IncRNA biomarkers associated with the progression of UCEC. (A) The variance rate of prediction accuracy for each of differentially expressed lncRNA. (B) The variance rate of classification performance when increasing numbers of the predictive lncRNAs. 
cohort and identified 625 mRNAs correlated with at least one of the five lncRNA biomarkers (Pearson correlation coefficient $>0.5$ and $\mathrm{p}<0.01)$. Then we performed functional enrichment analysis of mRNAs correlated with the five lncRNA biomarkers for Gene Ontology (GO) and Kyoto Encyclopedia of Genes and Genomes (KEGG). The results of GO analysis suggested that the 625 mRNAs clustered most significantly in three GO terms (including apoptotic signaling pathway, tumor necrosis factor-mediated signaling pathway and immune response) (Figure 5A) and four KEGG pathways (including p53 signaling pathway, Phosphatidylinositol signaling system and Viral carcinogenesis, Neurotrophin signaling pathway) (Figure 5B). These enriched functional categories are well known to be associated with the development and progression of cancer. Therefore, it is a plausible inference that dysregulated expression of five lncRNA biomarkers may lead to UCEC tumorigenesis and progress via regulating mRNAs involved in the known key cancerassociated pathway.

\section{DISCUSSION}

Endometrial carcinoma is the most common gynecologic malignancy. Standard management of endometrial cancer at diagnosis involves surgery, followed by chemotherapy with or without radiation therapy. Traditional histopathologic features, including histologic grade, tumor diameter, depth of myometrial invasion and status of lymphovascular space involvement,

A

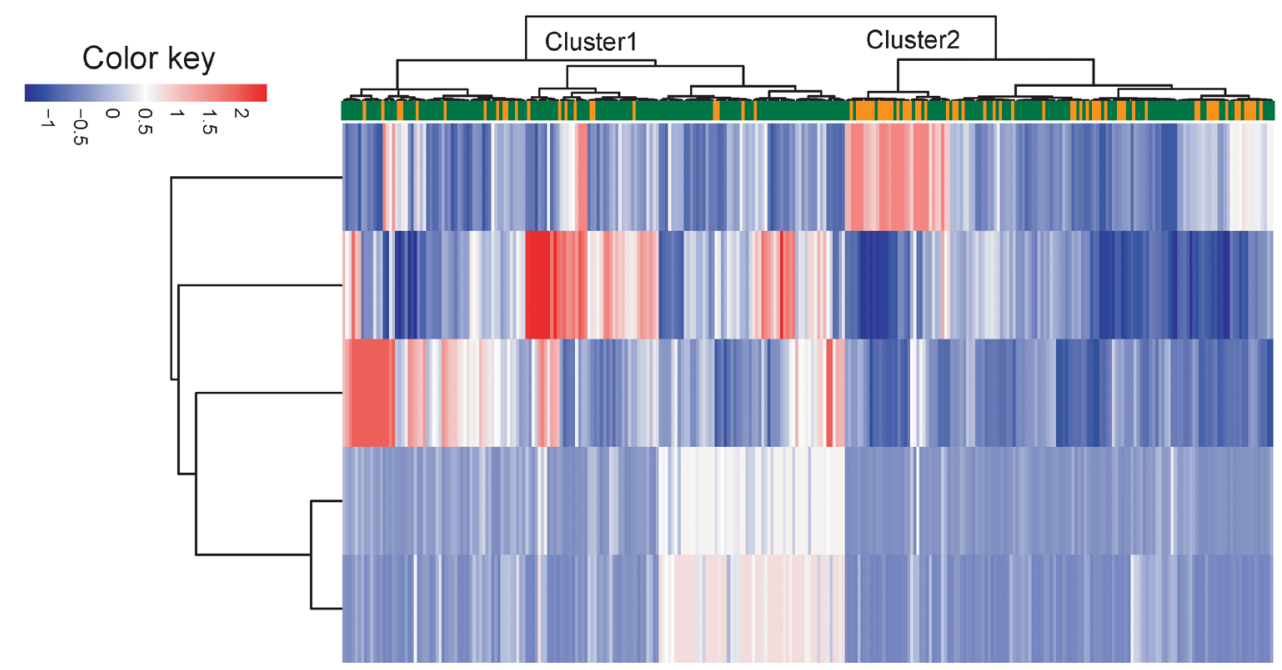

early stage

advanced stage

B

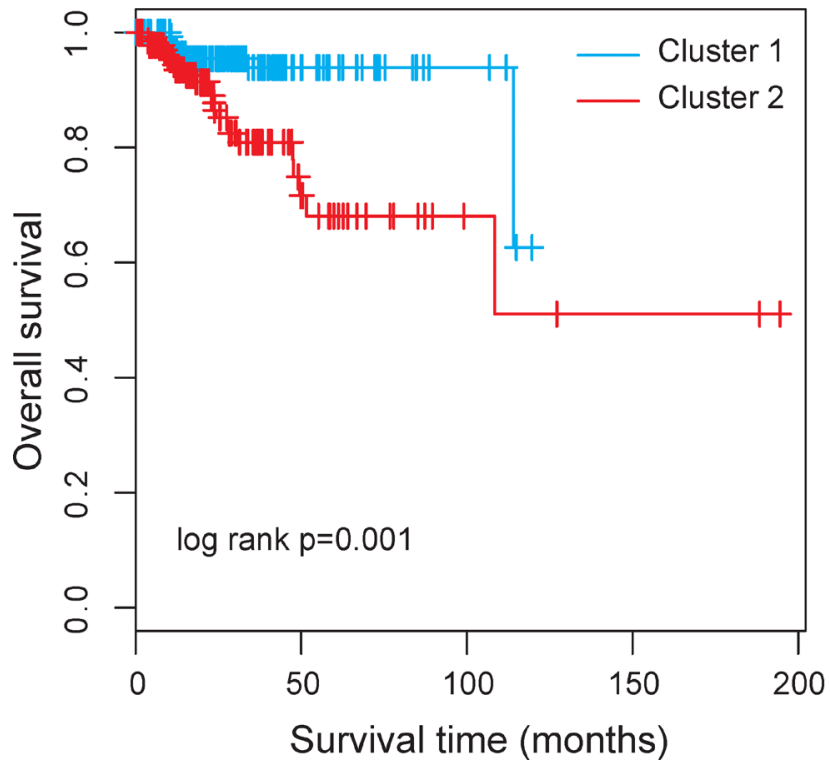

C

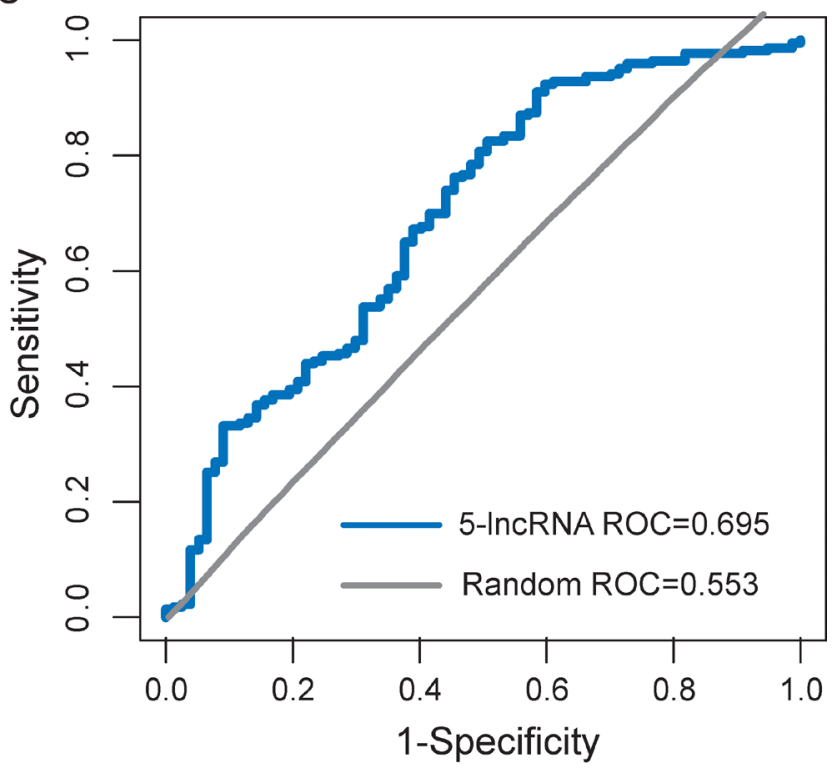

Figure 4: Performance evaluation of five IncRNA biomarkers in distinguishing advanced stage from early stage. (A) The unsupervised hierarchical clustering heatmap of 300 UCEC samples based on the expression profiles of five lncRNA biomarkers. (B) Kaplan-Meier survival curve for overall survival between the predicted two risk groups. (C) ROC analysis of five-lncRNA risk classifier using LOOCV. 
have been used to identify those at high-risk for disease progression and guide adjuvant treatment decisions [21]. Like many malignancies, UCEC is a complex disease characterized by both genetic, epigenetic and environmental factors [22]. The risk factors associated with disease relapse remain unclear. Because having no consideration of molecular heterogeneity, traditional histopathologic features are insufficient for making adjuvant treatment decisions [23]. Previous studies have focused on altered mRNA and miRNA expression and identified several molecular biomarkers for survival and recurrence prediction of endometrial carcinoma patients [21, 24-27]. Recently, dysregulated lncRNA expression has been implicated in the development and progression of tumors. Increasing evidence suggests that lncRNAs have an intrinsic advantage in their use as diagnostic or prognostic biomarkers compared to protein-coding genes and miRNAs since expression of lncRNA is a better indicator of the tumor status [28]. The emerging roles of lncRNAs in endometrial carcinoma have been investigated in several studies. For example, a study of 3 paired endometrial carcinoma and adjacent non-tumor tissues identified 53 differentially expressed lncRNAs and validated the potential function of IncRNA ASLNC04080 in endometrial carcinoma genesis and progression [29]. Another study performed by Xu et al. also identified 172 dysregulated lncRNAs by studying the expression profiles of IncRNA in EC as compared to normal endometrium [30]. Although the above studies revealed perturbed expression of IncRNAs in endometrial carcinoma, the research of diagnostic and prognostic value of lncRNAs is presently in its infancy.

In this study, we performed genome-wide analysis of 1377 lncRNAs in a large number of endometrial carcinoma patients from TCGA and found altered lncRNA expression patterns during the progression of UCEC, implying the potential roles of lncRNA as predictive biomarkers for

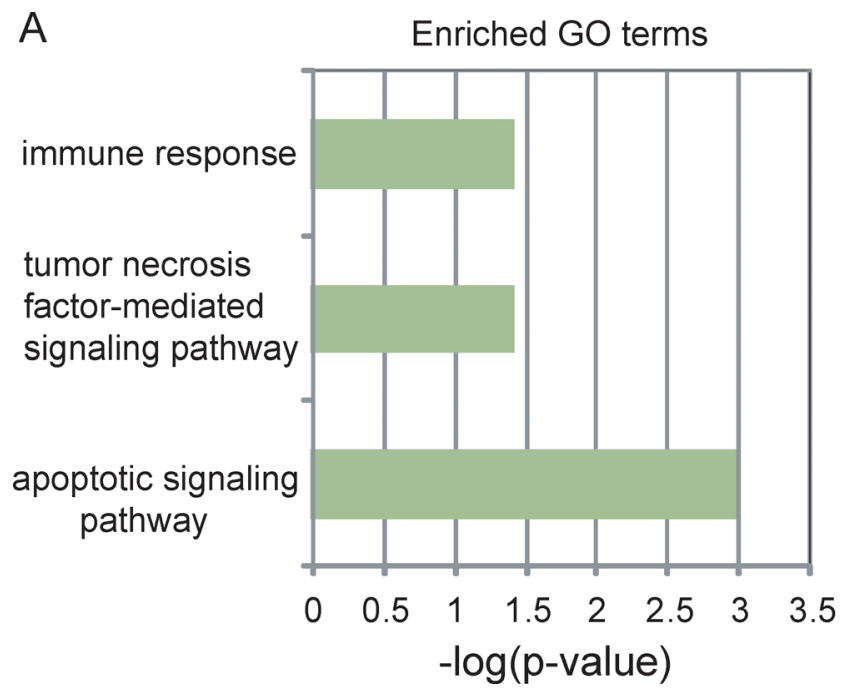

the discrimination of the high-risk endometrial carcinoma patients. In order to predict lncRNA biomarkers specific to endometrial carcinoma progression, we have searched for IncRNA combinations among the 17 differentially expressed lncRNAs, whose expression pattern may distinguish high-risk patients from those with earlystage using random forest feature selection which is necessary to avoid a small sample-per-feature ratio and provide better classification [31, 32]. A five-lncRNA combination, (FLJ27354, RP11-275I14.4, VIM-AS1, CTB-51J22.1 and RP11-229P13.20), has been identified as optimal biomarkers for EC progression. Then these five lncRNAs were integrated into a risk classifier using support vector machine and achieved a $78 \%$ prediction accuracy with $96.6 \%$ sensitivity and $76.6 \%$ specificity in stratifying early and later stages of endometrial carcinoma patients using LOOCV. As demonstrated in previous studies [33, 34], LOOCV has been widely recognized and increasingly used by investigators to examine the quality of various classifiers with SVM as the prediction engine. These findings demonstrated the feasibility and potential power of the five lncRNA biomarkers in identifying endometrial carcinoma patients at high risk for progression.

Although more and more lncRNAs have been identified, current knowledge for functional roles is relatively limited and only a few of lncRNAs have been well functionally characterized. Increasing evidence has suggested that lncRNAs function by regulating or interacting with its partner molecule. Therefore, it is widely used to associate specific lncRNAs with biological processes by correlating a common expression pattern of lncRNAs with protein-coding genes [28, 35]. Inspired by the above method, in order to investigate the functional roles of identified five lncRNA biomarkers in UCEC biology, we first identified protein-coding genes that are co-expressed with each lncRNA biomarker, and

B

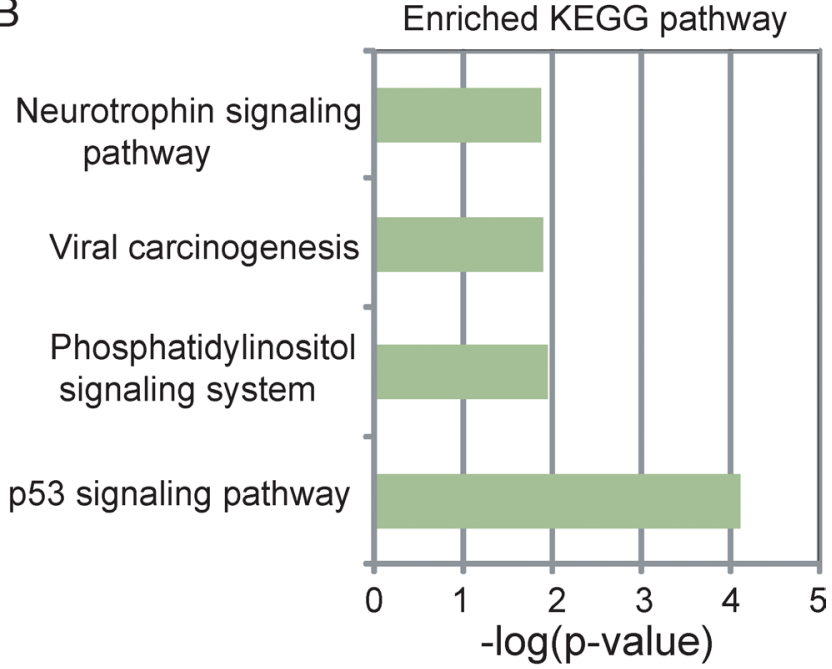

Figure 5: Functional prediction of five IncRNA biomarkers. (A) The enriched GO terms ranked by-log10 ( $p$-value). (B) The enriched KEGG pathways ranked by $-\log 10$ ( $p$-value). 
then performed guilt by association analysis to identify the potential function of lncRNAs by performing functional enrichment analysis for their co-expressed protein-coding genes. According to the above analysis, five lncRNA biomarkers were predicted to participate in several known cancer-related biological progress such as p53 signaling pathway, Phosphatidylinositol signaling system and Viral carcinogenesis, Neurotrophin signaling pathway. Previous studies have shown that aberrant P53 signaling pathways might play an important role in uterine and endometrial cancer [36]. In human endometrial carcinoma, p53 mutations the most frequent genetic events identified in aggressive nonendometrioid cancer [37]. Multiple links between the cellular phosphoinositide system and cancer have been observed [38]. For UCEC, Phosphatidylinositol 3-kinase signaling regulates insulin-like growth factor binding protein-3 expression in endometrial cancer cell lines [39]. Moreover, there were important interactions between the PI3K-AKT and p53 signaling pathways [40]. Neurotrophin signaling in the pathogenesis of cancer has been found to be associated with to stimulation of mitogenesis, promotion of metastasis and invasiveness, and inhibition of apoptosis [41]. These results of guilt by association analysis suggested that these five lncRNA biomarkers may play critical roles in the progression of UCEC by participating in important cancer-related biological processes.

In conclusion, our study has shown that the lncRNA expression profiles are altered in the advanced-stage UCEC patients compared with early-stage patients. We identified five novel lncRNA biomarkers that are significantly associated with the progression of UCEC by using random forest feature selection procedure, and developed a five-lncRNA risk classifier using SVM which significantly discriminate high-risk UCEC patients from persons with early stage with high performance. To our knowledge, it is the first investigation to identify lncRNA biomarkers for UCEC progression. Further validation studies in prospective datasets are needed to test the predictive power of the risk classifier before it is applied clinically.

\section{MATERIALS AND METHODS}

\section{Patient and clinical characteristics}

Clinical characteristics of 300 UCEC patients with stage information were obtained from The Cancer Genome Atlas (TCGA) project (https://cancergenome.nih.gov/). UCEC patients used in this study included 223 earlystage patients (207 patients with stage I and 16 patients with stage II) and 77 advanced-stage patients (64 patients with stage III and 13 patients with stage IV). The detailed clinical characteristics of 300 UCEC patients used in this study were summarized in Table 2.

\section{Genome-wide RNA-sequencing data of mRNAs and IncRNAs in UCEC patients}

Genome-wide lncRNA and mRNA expression of 300 UCEC patients were retrieved from TCGA long noncoding RNAs database (http://larssonlab.org/tcga-lncrnas/ index.php) according to Akrami et al. [42], including 10419 lncRNAs and 15977 mRNAs, respectively. Briefly, RNAseq data of TCGA UCEC patients in BAM format were realigned to the $\mathrm{Hg} 19$ assembly with TopHat and read counts for individual GENCODE genes were subsequently determined using HTSeq-count in "intersection-strict" mode, by considering only uniquely mapped reads. RPKM expression levels for lncRNAs and mRNAs were finally calculated by normalizing for lncRNA and mRNA length, and were $\log 2$ transformed. Then those lncRNAs with missing expression values in $>10 \%$ samples were filtered which resulted in 1377 lncRNAs for subsequent analysis.

\section{Analysis of IncRNA expression profiles}

Differential expression analysis by comparing lncRNA expression pattern in early-stage patients with those in advanced-stage patients using student $t$-test based on log-scale expression values. Differentially expressed lncRNAs were identified at the threshold of t-statistic $>4$ (or $<-4$ ) and false discovery rate $($ FDR) $<0.01$ (Benjamini and Hochberg algorithm). Hierarchical clustering of the expression values of differentially expressed lncRNAs was performed with R package "pheatmap" using the metric of Euclidean distance and complete linkage. The chi-square test was used to evaluate the significance between disease progression status and lncRNA biomarkers.

\section{Statistics for classification and prediction}

For classification of early-stage patients vs. advanced-stage patients, a support vector machine (SVM) was applied with the sigmoid kernel using $\mathrm{R}$ package "randomForest". An unbiased performance estimate in the classification of early-stage patients vs. advancedstage patients was performed using leave one out crossvalidation (LOOCV). Diagnostic ability of classification prediction was evaluated by obtaining the area under a receiver operating characteristic (ROC) curve (AUC) and diagnostic odds ratio (DOR). Kaplan-Meier survival plots and log-rank tests were used to assess the differences in patient outcomes between the predicted high-risk and lowrisk groups.

To identify optimal lncRNA biomarkers stratifying early and advanced stages of UCEC, we performed feature selection procedure as previously described [31]: (i) random forest importance value for each of differentially expressed lncRNA were obtained to represent the standardized drop in prediction accuracy. (ii) differentially expressed lncRNAs was re-ranked 
Table 2: Clinical covariates for the TCGA UCEC cohort

\begin{tabular}{|c|c|c|}
\hline Variables & & $\begin{array}{c}\text { Total TCGA } \\
\qquad N=\mathbf{3 0 0}\end{array}$ \\
\hline \multirow{4}{*}{ Stage, no $(\%)$} & $\mathrm{I}$ & $207(68.9)$ \\
\hline & II & $16(5.3)$ \\
\hline & III & $64(21.3)$ \\
\hline & IV & $13(4.3)$ \\
\hline \multirow{3}{*}{ Grade, no(\%) } & 1 & $70(23.3)$ \\
\hline & 2 & $80(26.7)$ \\
\hline & 3 & $150(50)$ \\
\hline \multirow{3}{*}{ histology, no(\%) } & Endometrioid & $242(80.7)$ \\
\hline & Serous & $50(16.7)$ \\
\hline & Mixed & $8(2.7)$ \\
\hline \multirow{2}{*}{ Vital status (\%) } & Alive & $269(89.7)$ \\
\hline & Dead & $31(10.3)$ \\
\hline Age, years, Mean (range) & & $63.4(62.1-64.6)$ \\
\hline
\end{tabular}

according to their random forest importance value. (iii) finding the optimal number of features by subsequently adding one lncRNA at a time in a top down forwardwrapper approach starting with the top two lncRNAs of the ranked list; at each increment, the DOR was assessed using LOOCV.

\section{Functional enrichment analysis}

Functional enrichment analysis of GO and KEGG was performed using DAVID Bioinformatics Tool (version 6.7) [72] limited to GO terms in the "Biological Process"(GOTERM-BP-FAT) and KEGG pathway categories. The biological processes and pathways with $p$-value of $<0.05$ using the whole human genome as background were considered as significant enriched functional categories.

\section{CONFLICTS OF INTEREST}

The authors declare that they have no of interest.

\section{REFERENCES}

1. Sonoda Y, Barakat RR. Screening and the prevention of gynecologic cancer: endometrial cancer. Best Pract Res Clin Obstet Gynaecol. 2006; 20:363-377.

2. Gottwald L, Pluta P, Piekarski J, Spych M, Hendzel K, Topczewska-Tylinska K, Nejc D, Bibik R, Korczynski J, Cialkowska-Rysz A. Long-term survival of endometrioid endometrial cancer patients. Arch Med Sci. 2010; 6:937-944.

3. Kupets R, Le T, SOGC-GOC-SCC POLICY AND PRACTICE GUIDELINES COMMITTEE, and SPECIAL CONTRIBUTORS. The role of adjuvant therapy in endometrial cancer. J Obstet Gynaecol Can. 2013; 35:375-379.
4. Derrien T, Johnson R, Bussotti G, Tanzer A, Djebali S, Tilgner H, Guernec G, Martin D, Merkel A, Knowles DG, Lagarde J, Veeravalli L, Ruan X, et al. The GENCODE v7 catalog of human long noncoding RNAs: analysis of their gene structure, evolution, and expression. Genome Res. 2012; 22:1775-1789.

5. ENCODE Project Consortium, Birney E, Stamatoyannopoulos JA, Dutta A, Guigo R, Gingeras TR, Margulies EH, Weng Z, Snyder M, Dermitzakis ET, Thurman RE, Kuehn MS, Taylor $\mathrm{CM}$, et al. Identification and analysis of functional elements in $1 \%$ of the human genome by the ENCODE pilot project. Nature. 2007; 447:799-816.

6. Ponting CP, Oliver PL, Reik W. Evolution and functions of long noncoding RNAs. Cell. 2009; 136:629-641.

7. Rinn JL, Chang HY. Genome regulation by long noncoding RNAs. Annu Rev Biochem. 2012; 81:145-166.

8. Mercer TR, Mattick JS. Structure and function of long noncoding RNAs in epigenetic regulation. Nat Struct Mol Biol. 2013; 20:300-307.

9. Kornienko AE, Guenzl PM, Barlow DP, Pauler FM. Gene regulation by the act of long non-coding RNA transcription. BMC Biol. 2013; 11:59.

10. Hangauer MJ, Vaughn IW, McManus MT. Pervasive transcription of the human genome produces thousands of previously unidentified long intergenic noncoding RNAs. PLoS Genet. 2013; 9:e1003569.

11. Chen D, Sun Q, Cheng X, Zhang L, Song W, Zhou D, Lin J, Wang W. Genome-wide analysis of long noncoding RNA (lncRNA) expression in colorectal cancer tissues from patients with liver metastasis. Cancer Med. 2016; 5:1629-1639.

12. Dong R, Jia D, Xue P, Cui X, Li K, Zheng S, He X, Dong K. Genome-wide analysis of long noncoding RNA (lncRNA) expression in hepatoblastoma tissues. PLoS One. 2014; 9:e85599. 
13. Zhou M, Sun Y, Xu W, Zhang Z, Zhao H, Zhong Z, Sun J. Comprehensive analysis of lncRNA expression profiles reveals a novel lncRNA signature to discriminate nonequivalent outcomes in patients with ovarian cancer. Oncotarget. 2016; 7:32433-32448. doi: 10.18632/oncotarget.8653.

14. Zhou M, Xu W, Yue X, Zhao H, Wang Z, Shi H, Cheng L, Sun J. Relapse-related long non-coding RNA signature to improve prognosis prediction of lung adenocarcinoma. Oncotarget. 2016; 7:29720-29738. doi: 10.18632/oncotarget.8825.

15. Zhou M, Wang X, Shi H, Cheng L, Wang Z, Zhao H, Yang L, Sun J. Characterization of long non-coding RNA-associated ceRNA network to reveal potential prognostic lncRNA biomarkers in human ovarian cancer. Oncotarget. 2016; 7:12598. doi: 10.18632/oncotarget.7181.

16. Zhou M, Zhao H, Xu W, Bao S, Cheng L, Sun J. Discovery and validation of immune-associated long non-coding RNA biomarkers associated with clinically molecular subtype and prognosis in diffuse large B cell lymphoma. Mol Cancer. 2017; $16: 16$.

17. Nana-Sinkam SP, Croce CM. Non-coding RNAs in cancer initiation and progression and as novel biomarkers. Mol Oncol. 2011; 5:483-491.

18. Spizzo R, Almeida MI, Colombatti A, Calin GA. Long noncoding RNAs and cancer: a new frontier of translational research? Oncogene. 2012; 31:4577-4587.

19. Cheetham SW, Gruhl F, Mattick JS, Dinger ME. Long noncoding RNAs and the genetics of cancer. Br J Cancer. 2013; 108:2419-2425.

20. Prensner JR, Chinnaiyan AM. The emergence of lncRNAs in cancer biology. Cancer Discov. 2011; 1:391-407.

21. Levan K, Partheen K, Osterberg L, Olsson B, Delle U, Eklind S, Horvath G. Identification of a gene expression signature for survival prediction in type I endometrial carcinoma. Gene Expr. 2010; 14:361-370.

22. O'Hara AJ, Bell DW. The genomics and genetics of endometrial cancer. Adv Genomics Genet. 2012; 2012:33-47.

23. Zhang F, Ren C, Zhao H, Yang L, Su F, Zhou MM, Han J, Sobie EA, Walsh MJ. Identification of novel prognostic indicators for triple-negative breast cancer patients through integrative analysis of cancer genomics data and protein interactome data. Oncotarget. 2016; 7:71620-71634. doi: 10.18632/oncotarget.12287.

24. Zhai H, Karaayvaz M, Dong P, Sakuragi N, Ju J. Prognostic significance of miR-194 in endometrial cancer. Biomark Res. 2013; 1.

25. Torres A, Torres K, Pesci A, Ceccaroni M, Paszkowski T, Cassandrini P, Zamboni G, Maciejewski R. Diagnostic and prognostic significance of miRNA signatures in tissues and plasma of endometrioid endometrial carcinoma patients. Int J Cancer. 2013; 132:1633-1645.

26. Ratner ES, Tuck D, Richter C, Nallur S, Patel RM, Schultz V, Hui P, Schwartz PE, Rutherford TJ, Weidhaas JB. MicroRNA signatures differentiate uterine cancer tumor subtypes. Gynecol Oncol. 2010; 118:251-257.
27. Karlsson S, Olsson B, Klinga-Levan K. Gene expression profiling predicts a three-gene expression signature of endometrial adenocarcinoma in a rat model. Cancer Cell Int. 2009; 9:12.

28. Huarte M. The emerging role of 1 ncRNAs in cancer. Nat Med. 2015; 21:1253-1261.

29. Zhai W, Li X, Wu S, Zhang Y, Pang H, Chen W. Microarray expression profile of IncRNAs and the upregulated ASLNC04080 lncRNA in human endometrial carcinoma. Int J Oncol. 2015; 46:2125-2137.

30. Xu J, Qian Y, Ye M, Fu Z, Jia X, Li W, Xu P, Lv M, Huang L, Wang L, Ruan H, Lv J. Distinct expression profile of lncRNA in endometrial carcinoma. Oncol Rep. 2016; 36:3405-3412.

31. Sorensen KP, Thomassen M, Tan Q, Bak M, Cold S, Burton M, Larsen MJ, Kruse TA. Long non-coding RNA expression profiles predict metastasis in lymph node-negative breast cancer independently of traditional prognostic markers. Breast Cancer Res. 2015; 17:55.

32. Somorjai RL, Dolenko B, Baumgartner R. Class prediction and discovery using gene microarray and proteomics mass spectroscopy data: curses, caveats, cautions. Bioinformatics. 2003; 19:1484-1491.

33. Liu B, Fang L, Long R, Lan X, Chou KC. iEnhancer2L: a two-layer predictor for identifying enhancers and their strength by pseudo k-tuple nucleotide composition. Bioinformatics. 2016; 32:362-369.

34. Liu B, Liu F, Wang X, Chen J, Fang L, Chou KC. Pse-inOne: a web server for generating various modes of pseudo components of DNA, RNA, and protein sequences. Nucleic Acids Res. 2015; 43:W65-71.

35. Ma H, Hao Y, Dong X, Gong Q, Chen J, Zhang J, Tian W. Molecular mechanisms and function prediction of long noncoding RNA. ScientificWorldJournal. 2012; 2012:541786.

36. Mhawech-Fauceglia P, Wang D, Kesterson J, Syriac S, Clark K, Frederick PJ, Lele S, Liu S. Gene expression profiles in stage I uterine serous carcinoma in comparison to grade 3 and grade 1 stage I endometrioid adenocarcinoma. PLoS One. 2011; 6:e18066.

37. Bansal N, Yendluri V, Wenham RM. The molecular biology of endometrial cancers and the implications for pathogenesis, classification, and targeted therapies. Cancer Control. 2009; 16:8-13.

38. Bunney TD, Katan M. Phosphoinositide signalling in cancer: beyond PI3K and PTEN. Nat Rev Cancer. 2010; 10:342-352.

39. Qiao M, Shapiro P, Kumar R, Passaniti A. Insulin-like growth factor-1 regulates endogenous RUNX2 activity in endothelial cells through a phosphatidylinositol 3-kinase/ ERK-dependent and Akt-independent signaling pathway. J Biol Chem. 2004; 279:42709-42718.

40. Catasus L, Gallardo A, Cuatrecasas M, Prat J. Concomitant PI3K-AKT and p53 alterations in endometrial carcinomas 
are associated with poor prognosis. Mod Pathol. 2009; 22:522-529.

41. Molloy NH, Read DE, Gorman AM. Nerve growth factor in cancer cell death and survival. Cancers (Basel). 2011; 3:510-530.
42. Akrami R, Jacobsen A, Hoell J, Schultz N, Sander C, Larsson E. Comprehensive analysis of long non-coding RNAs in ovarian cancer reveals global patterns and targeted DNA amplification. PLoS One. 2013; 8:e80306. 